\title{
Evaluación de la tolerancia a estrés por sequía en cuatro genotipos naturalizados de vid (Vitis vinifera) provenientes del norte de Chile
}

\author{
Evaluation of drought stress tolerance in four naturalized grapevine genotypes \\ (Vitis vinifera) from northern Chile \\ Claudia Bavestrello-Riquelme ${ }^{1,5}$, Loreto Cavieres ${ }^{1}$, Jorge Gallardo ${ }^{1}$, Antonio Ibacache ${ }^{1,2}$, \\ Nicolás Franck ${ }^{3,4}$, Andrés Zurita-Silva, *
}

\begin{abstract}
RESUMEN
El cultivo de la vid (Vitis vinifera) se caracteriza por sufrir sequías estacionales en la mayoría de las regiones productoras, situación que se incrementa en ambientes áridos y semiáridos de Chile, los cuales han sufrido disminuciones de precipitaciones y prolongados periodos de sequía, siendo una de las principales problemáticas que afectan la productividad de las vides en el norte del país. El objetivo planteado fue analizar las respuestas morfo-fisiológicas en genotipos naturalizados de vides expuestas a estrés hídrico y determinar su nivel de tolerancia. Se evaluaron parámetros fisiológicos y arquitecturales en cuatro genotipos de vides (10, 93, $134,148)$, provenientes de un gradiente latitudinal en zonas áridas y semiáridas del norte de Chile $\left(18^{\circ} \mathrm{S}\right.$ a $\left.32^{\circ} \mathrm{S}\right)$. Mediante el uso de un sistema de mini-rizotrones, material clonal de los cuatro genotipos fue sometido a tratamientos de estrés hídrico leve y severo (60\% y $40 \%$ de capacidad de campo, respectivamente), durante tres meses, bajo un diseño completamente aleatorio. Se obtuvieron diferencias significativas a nivel de tratamientos para la sobrevivencia y longitud de tallo, distancia entrenudos y todos los parámetros de desempeño fisiológico evaluados. Asimismo, se obtuvieron diferencias significativas entre los genotipos en caracteres de longitud de raíz, distancia entrenudos, fotosíntesis y potencial hídrico. Ello permitió identificar genotipos anhisohídricos como 134 y 148, de genotipos isohídricos, como 10 y 93 en relación al comportamiento de respuesta al estrés. La determinación de efectos tempranos de la sequía en desarrollo, sobrevivencia, y desempeño fisiológico, permitió diferenciar al genotipo 134 como el más tolerante de los evaluados. La metodología utilizada permitirá seleccionar nuevos genotipos para uso potencial como portainjertos o injertos en zonas áridas y semiáridas, subrayando el rol fundamental de explorar la variabilidad genética de vides y su uso como cultivo modelo.
\end{abstract}

Palabras clave: estrés hídrico, desarrollo radical, respuestas fisiológicas, Vitis vinifera.

\begin{abstract}
The grapevine (Vitis vinifera) is grown and characterized by seasonal drought suffered in most of the world's producing regions, a situation that increases in arid and semiarid regions of Chile, which have experienced declines in rainfall and prolonged dry periods, one of the main problems that affect the productivity of the vines in these regions. The research aim was to analyze the morpho-physiological responses of naturalized grapevine genotypes exposed to water stress and determine their level of tolerance. Physiological and architectural parameters were evaluated in four grapevine genotypes (10, 93, 134, 148), collected from a latitudinal gradient in arid and semi-arid northern Chile $\left(18^{\circ} \mathrm{SL}\right.$ to $\left.32^{\circ} \mathrm{SL}\right)$. By using a system of mini-rhizotrons, clonal material of the four genotypes was subjected to mild and severe (60\% and $40 \%$ field capacity, respectively), water stress treatments during three months under a fully randomized design. Significant differences were obtained at treatment's level for survival and stem length, internode distance and all physiological performance parameters evaluated. Furthermore, there were significant differences between genotypes in traits such as root length, internode distance, photosynthesis and water potential. This allowed identifying anisohydric genotypes such as 134 and 148, from near isohydric genotypes, such as 10 and 93 in relation to the stress response behaviour. Determination of early drought effects in development, survival, and physiological performance, allowed differentiating genotype 134 as the most tolerant of those assessed. This methodology will enable to select new genotypes for potential use as rootstocks or grafts for arid and semi-arid regions, emphasizing the fundamental role of exploring the grapevine genetic variability and its use as a crop model.
\end{abstract}

Key words: water stress, root development, physiological performance, Vitis vinifera..

1 Centro de Estudios Avanzados en Zonas Áridas (CEAZA), Universidad de La Serena, Av. Raúl Bitrán s/n, La Serena, Chile. 2 Instituto de Investigaciones Agropecuarias INIA, Centro de Investigación Intihuasi, Colina San Joaquin s/n, La Serena, Chile. 3 Centro de Estudios de Zonas Áridas, Facultad de Ciencias Agronómicas, Universidad de Chile, Casilla 129, Coquimbo, Chile. 4 Departamento de Producción Agrícola, Facultad de Ciencias Agronómicas, Universidad de Chile. Casilla 1004, Santiago, Chile.

5 Facultad de Ciencias Agropecuarias y Forestales, Universidad de La Frontera. Av. Francisco Salazar 1145, Temuco, Chile.

* Corresponding author. Centro de Estudios Avanzados en Zonas Áridas (CEAZA), Universidad de La Serena, Av. Raúl Bitrán s/n, PO Box 554, La Serena, Chile. E-mail address: andres.zurita@ceaza.cl. Tel +5651204378; fax +5651334741.

Fecha de Recepción: 12 Julio, 2012.

Fecha de Aceptación: 4 Octubre, 2012. 


\section{Introducción}

En Chile, la producción de vides se realiza entre las regiones de Arica-Parinacota y Los Lagos, siendo el principal frutal de exportación de Chile al mundo, con un $16,9 \%$ del total de las exportaciones frutícolas (ODEPA, 2012). Existen factores ambientales desfavorables a los cuales se ven enfrentadas las vides cultivadas en ambientes áridos y semiáridos, como son la sequía y la salinidad de los suelos. El agua es uno de los factores más importantes para el desarrollo de las plantas y su carencia constituye una de las principales fuentes de estrés (Chaves et al., 2009). Especies como la vid han desarrollado diversas respuestas adaptativas que les permiten tolerar diferentes niveles de restricción hídrica, permitiéndoles completar su desarrollo (Rogiers et al., 2012).

Las vides se caracterizan por sufrir sequías estacionales en la mayoría de las regiones productoras (Grimplet et al., 2008; Chaves et al., 2010), y su diversidad genotípica en la tolerancia a la sequía convierte a este cultivo perenne en un modelo de estudio ideal, dada la existencia de respuestas al déficit hídrico que son genotipo-dependiente, gracias a la gran variabilidad genética existente en las numerosas variedades comerciales disponibles y materiales naturalizados en las zonas de origen y cultivo (Bota et al., 2001; Gaudillère et al., 2002; Chaves et al., 2010; Rogiers et al., 2012). Respuestas comunes al estrés incluyen cambios tanto en el metabolismo de hormonas, como en procesos de fotosíntesis, crecimiento, transcripción y señalización entre otros, los cuales han sido confirmados mediante análisis fisiológicos y moleculares (revisados por Cramer, 2010). En particular, el ácido abscísico (ABA) afecta las rutas de señalización que disparan actividades regulatorias claves del metabolismo y transcripción, y también regulan importantes respuestas fisiológicas tales como la conductancia estomática, fotoprotección y desarrollo (Chaves et al., 2010; Cramer, 2010). Otro de los mecanismos de tolerancia a sequía es el incremento en sistemas radiculares ramificados y profundos, capaces de obtener suministros de agua para su desarrollo y así sobrevivir a períodos de déficit hídrico (Chaves et al., 2010; Lovisolo et al., 2010; Rogiers et al., 2012). Además, la vid presenta mecanismos para evitar el déficit hídrico, los cuales incluyen el incrementar al máximo la eficiencia en el uso del agua a través de la regulación estomática, incluso con leves cambios en el potencial hídrico foliar (Flexas et al., 1998; Lovisolo et al., 2010). Otro mecanismo es el ajuste osmótico mediante la acumulación activa de azúcares solubles, ácidos orgánicos y prolina (Castellarin et al., 2007; Deluc et al., 2009). Esto permite mantener la turgencia celular a bajos potenciales hídricos (Morgan, 1984), facilitando el crecimiento celular, la apertura estomática y la fotosíntesis, favoreciendo tanto la supervivencia como las adaptaciones radiculares para explorar un mayor volumen de agua del suelo (Turner y Jones, 1980; Blum et al., 1989; Schultz, 2003). Basado en el comportamiento del potencial hídrico en respuesta al déficit hídrico, los cultivares de vid han sido clasificados como isohídricos o anisohídricos (Schultz, 2003; Vandeleur et al., 2009). Cultivares isohídricos son aquellos que mantienen su potencial hídrico foliar sobre un determinado umbral, a pesar de la disponibilidad hídrica de suelo o la demanda hídrica atmosférica. En tanto, cultivares anisohídricos son aquellos en los cuales el potencial hídrico foliar cae con la disminución en la disponibilidad hídrica de suelo o con incrementos en la demanda hídrica atmosférica (revisados por Lovisolo et al., 2010). En vides isohídricas, el potencial hídrico foliar raramente cae bajo -1,5 MPa, lo cual es cercano al umbral de cavitación severa en esta especie (Lovisolo et al., 2010).

El objetivo del presente estudio fue analizar las respuestas morfo-fisiológicas en genotipos naturalizados de vides expuestas a estrés hídrico. Específicamente, se determinaron los cambios que sufren estas plantas para adaptarse al estrés por sequía mediante la evaluación de parámetros fisiológicos y arquitecturales en cuatro genotipos de vides, provenientes de zonas áridas y semiáridas del norte de Chile, mediante el uso de un sistema controlado de minirizotrones.

\section{Materiales y Métodos}

\section{Material vegetal}

Se analizaron cuatro genotipos de vides colectados en la zona norte de Chile, los cuales fueron seleccionados por el mayor crecimiento al ser sometidos a condiciones de cultivo in vitro: genotipos 148 y 134, colectados en la Región de Tarapacá; genotipo 10, colectado en la Región de Atacama; y genotipo 93, colectado en la Región de Coquimbo.

Se utilizó material clonal de cinco meses, proveniente de cultivo in vitro. El proceso de 
micropropagación consistió en tres etapas de un mes cada una: establecimiento, crecimiento y enraizamiento, utilizando medio MS modificado (Murashige and Skoog, 1962). Las plántulas de tres meses permanecieron en un cuarto de crecimiento en condiciones controladas y constantes (16 horas de luz, temperatura promedio de $24^{\circ} \mathrm{C}$ y humedad relativa $70 \%$ ). Una vez que las plantas presentaron raíces in vitro, se procedió a su trasplante y aclimatación por un mes adicional en un sustrato de turba: perlita (1:1), regadas con una solución nutritiva de MS al 0,5X. Durante la fase de aclimatación las plantas fueron trasplantadas a un contenedor cerrado, el cual se fue adaptando gradualmente a condiciones ex vitro, durante cuatro semanas en cuarto de crecimiento. El proceso de aclimatación en invernadero fue de cuatro semanas adicionales, donde fueron regadas con una solución nutritiva MS al 0,1X semanalmente. Finalmente, las plantas de cinco meses fueron instaladas en minirizotrones, conformados por dos vidrios de 37 x $30 \mathrm{~cm}$ con sustrato de turba: perlita (1:1), cubierto por ambos lados con una lámina oscura para evitar la exposición a la luz del sistema radicular. Se instalaron dos plantas del mismo genotipo, separadas por una banda de hule para evitar que sus raíces se entrelazaran. Tres minirizotrones se instalaron en contenedores plásticos cerrados, separados por una distancia de $4 \mathrm{~cm}$ entre ellos. Las plantas crecieron durante una semana antes de comenzar con la aplicación de los diferentes tratamientos de riego.

\section{Tratamiento de estrés por sequía}

Para determinar la cantidad de riego a utilizar se realizó, previamente, un ensayo para registrar el contenido relativo de agua del sustrato en los minirizotrones durante 35 días, el cual consistió en dos tratamientos de riego, un control con riego semanal a capacidad de campo y un tratamiento de sequía absoluta, monitoreados por sondas ECH2O (EC-20, USA). Esto permitió determinar que la cantidad de agua suficiente para mantener el rizotrón a capacidad de campo (CC) fue de 55 $\mathrm{ml}$ por semana. El ensayo se realizó mediante dos tratamientos de riego deficitario: estrés hídrico leve (60\% de CC) y estrés hídrico severo (40\% de $\mathrm{CC}$ ), y su control respectivo (CC), con un diseño completamente aleatorizado. Por cada genotipo se analizaron 18 plantas, divididas en tres tratamientos con seis repeticiones por tratamiento.

\section{Parámetros evaluados}

El experimento finalizó cumplidos tres meses, y se midieron la supervivencia de las plantas y longitud de raíz final $(\mathrm{cm})$ en los tratamientos de estrés hídrico y el control. La evaluación morfométrica se realizó cada 15 días, midiendo la distancia entre nudos $(\mathrm{cm})$ y longitud de tallo $(\mathrm{cm})$. Las mediciones de desempeño fisiológico como tasa de fotosíntesis se realizaron cada 15 días sobre tres hojas visualmente sanas y totalmente expuestas al sol, mediante un equipo de intercambio gaseoso IRGA (Licor-6400, USA). El potencial hídrico foliar $\left(\Psi_{\mathrm{h}}\right)$ se evaluó cada 15 días mediante un psicrómetro y una termocupla, lo que permitió determinar la evapotranspiración de la hoja mediante el principio de Peltier (Busso, 2008). La tasa de crecimiento relativo se calculó tomando el crecimiento final versus el inicial en relación al número de mediciones. Todas las mediciones fueron realizadas a las 12:00 hrs. Considerando que no hubo diferencias entre mediciones en los datos temporales (datos no mostrados), se presentan solamente los datos del final del experimento.

Los parámetros morfométricos así como capacidad fotosintética, potencial hídrico y tasa de crecimiento relativo fueron analizados mediante un análisis de varianza (ANOVA) factorial. Previamente se evaluaron la normalidad de los datos y la homogeneidad de la varianza de acuerdo al test Shapiro-Wilks y Bartlett, respectivamente, y las diferencias entre tratamientos fueron evaluadas mediante la prueba a posteriori de Tukey. La variable de sobrevivencia fue analizada con un modelo lineal generalizado debido a que los datos no cumplían los principales supuestos de normalidad y homocedasticidad, y la regresión aplicada para este análisis fue Poisson (Sokal y Rohlf, 1995).

\section{Resultados}

\section{Sobrevivencia de plantas}

La sobrevivencia de plantas disminuyó de manera significativa por efecto del tratamiento de estrés hídrico, siendo el tratamiento de estrés hídrico severo el que disminuyó la sobrevivencia de los genotipos hasta un $20 \%$, a excepción del genotipo 134 , en donde cerca de un $50 \%$ de las plantas sobrevivieron a este nivel de estrés (Tabla 1; Figura 1). No existió efecto significativo entre los genotipos ni en la interacción entre genotipo y tratamiento (Tabla 1). 
Tabla 1. Parámetros estadísticos para la fuente de variación (tratamiento, genotipo, interacción tratamiento * genotipo), medida en sobrevivencia y parámetros morfométricos de cuatro genotipos diferentes de vid bajo tratamientos de estrés hídrico.

\begin{tabular}{|c|c|c|c|c|c|c|c|c|c|c|c|c|}
\hline \multirow{2}{*}{ Fuente de variación } & \multicolumn{3}{|c|}{ Sobrevivencia } & \multicolumn{3}{|c|}{ Longitud de tallo } & \multicolumn{3}{|c|}{ Longitud de raíz } & \multicolumn{3}{|c|}{ Distancia entrenudos } \\
\hline & $\mathrm{gl}$ & Wald & $\mathrm{p}$ & $\mathrm{gl}$ & $\mathrm{F}$ & $\mathrm{p}$ & $\mathrm{gl}$ & $\mathrm{F}$ & $\mathrm{p}$ & $\mathrm{gl}$ & $\mathrm{F}$ & $\mathrm{p}$ \\
\hline Tratamiento $(\mathrm{T})$ & 2 & 8,220885 & 0,016401 & 2 & 29,2184 & 0,000000 & 2 & 2,502 & 0,090454 & 2 & 6,411 & 0,002996 \\
\hline Genotipo (G) & 3 & 0,605265 & 0,895226 & 3 & 1,5507 & 0,210755 & 3 & 4,615 & 0,005696 & 3 & 17,718 & 0,000000 \\
\hline $\mathrm{T}^{*} \mathrm{G}$ & 6 & 0,821608 & 0,991482 & 6 & 7,8563 & 0,000003 & 6 & 3,804 & 0,002811 & 6 & 1,749 & 0,125284 \\
\hline
\end{tabular}

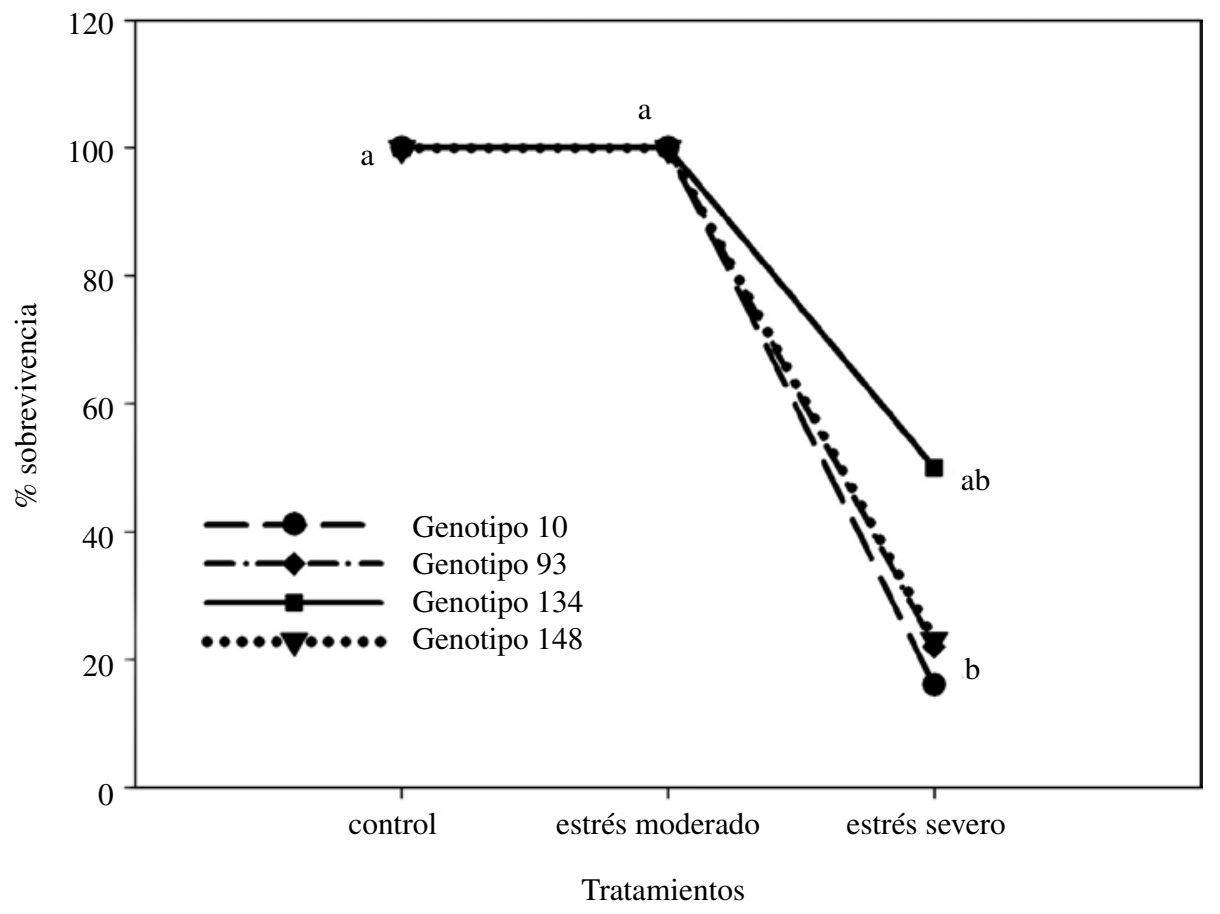

Figura 1. Sobrevivencia promedio $(\%)$ de cuatro genotipos naturalizados de Vitis vinifera expuestos a tratamiento control, estrés hídrico moderado y severo durante tres meses (Letras diferentes corresponden a diferencias significativas; $\mathrm{p}=0,05)$.

Survival (\%) in four naturalized genotypes of Vitis vinifera exposed to control, moderate and severe drought stress treatments during three months (different letters indicate significant differences; $p=0.05$ ).

\section{Parámetros morfométricos}

Las evaluaciones de longitud de tallo presentaron una disminución significativa entre los tratamientos aplicados, sin observarse diferencias significativas entre los genotipos (Tabla 1). El análisis evidenció un efecto significativo en el factor de interacción tratamiento por genotipo (Tabla 1). Las mayores diferencias se registraron entre tratamiento control y estrés severo, en donde los genotipos ajustaron su crecimiento a una longitud similar (Figura 2A). De hecho, las mayores diferencias en longitud entre los genotipos se presentaron sólo en tratamiento control entre los genotipos $148(40 \mathrm{~cm})$ y $10(10 \mathrm{~cm})$.

En relación a la longitud final de las raíces en los genotipos evaluados, este parámetro mostró una disminución significativa dependiente del genotipo y en la interacción genotipo por tratamiento, no así por el efecto del tratamiento (Tabla 1). Las diferencias entre los genotipos y los tratamientos se presentan en la Figura 2B, en donde los genotipos 10 y 134 presentaron las menores disminuciones de longitud 

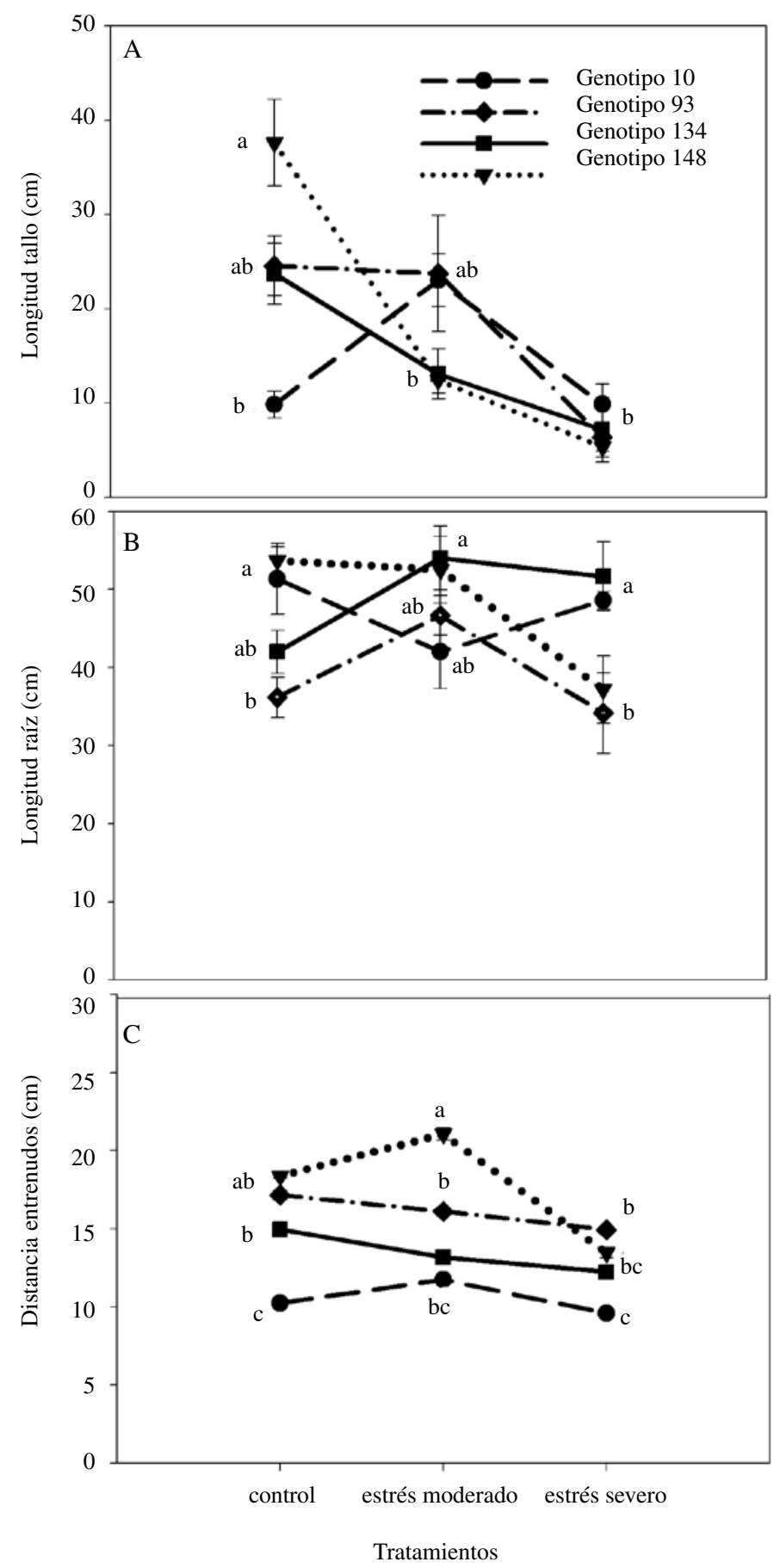

Figura 2. Longitud tallo (A), longitud de raíz (B) y distancia entrenudos (C), medidas en centímetros, evaluada en 4 genotipos diferentes de Vitis vinifera, en condiciones control, estrés hídrico moderado y severo durante tres meses. Se indican los valores promedio ( $\pm 2 \mathrm{SE}$ ). Letras diferentes corresponden a diferencias significativas; test de Tukey, $\alpha<0,05$.

Shoot length $(A)$, root length $(B)$ and internode distance $(C)$, measured in centimeters, assessed in 4 different genotypes of Vitis vinifera, in control conditions, moderate and severe drought stress during three months. Mean values ( $\pm 2 S E)$ are shown. Different letters indicate significant differences; Tukey test, $\alpha<0.05$. 
Tabla 2. Parámetros estadísticos para la fuente de variación (tratamiento, genotipo, interacción tratamiento * genotipo), medida en atributos de desempeño fisiológico de cuatro genotipos diferentes de vid bajo tratamientos de estrés hídrico.

\begin{tabular}{|c|c|c|c|c|c|c|c|c|c|}
\hline \multirow{2}{*}{ Fuente de variación } & \multicolumn{3}{|c|}{ Fotosíntesis } & \multicolumn{3}{|c|}{ Potencial hídrico foliar } & \multicolumn{3}{|c|}{ Tasa crecimiento relativo } \\
\hline & $\mathrm{gl}$ & $\mathrm{F}$ & $\mathrm{p}$ & $\mathrm{gl}$ & $\mathrm{F}$ & $\mathrm{p}$ & $\mathrm{gl}$ & $\mathrm{F}$ & $\mathrm{p}$ \\
\hline Tratamiento $(\mathrm{T})$ & 2 & 20,1723 & 0,000007 & 2 & 378,7325 & 0,000000 & 2 & 29,2184 & 0,000000 \\
\hline Genotipo (G) & 3 & 4,2443 & 0,015338 & 3 & 43,3690 & 0,000000 & 3 & 1,5507 & 0,210755 \\
\hline $\mathrm{T}^{*} \mathrm{G}$ & 6 & 1,2521 & 0,315857 & 6 & 43,3690 & 0,000000 & 6 & 7,8563 & 0,000003 \\
\hline
\end{tabular}

radical en respuesta al estrés severo, y los genotipos 93 y 148 presentaron la mayor disminución. La mayor longitud radical se observó en el genotipo 148 en el tratamiento control, pero, a medida que el nivel de estrés aumentó, dicho genotipo presentó la menor longitud radical. En cambio, el genotipo 134 incrementó su longitud radical tanto en estrés moderado como severo (Figura 2B).

La distancia entrenudos disminuyó de manera significativa entre los tratamientos, así como se presentaron diferencias significativas entre genotipos (Tabla 1). La mayor diferencia se registró entre los tratamientos moderado y severo, disminuyendo la distancia entrenudos. Entre los genotipos, la mayor distancia entrenudos se observó en los genotipos 93 y 148 y la menor distancia en el genotipo 10 en todos los tratamientos; entre los genotipos 134 y 148 no existieron diferencias significativas en el tratamiento de estrés severo (Figura 2C).

\section{Desempeño fisiológico}

La tasa fotosintética medida a radiación saturante de $750 \mu \mathrm{mol}\left(\mathrm{A}_{\max }\right)$ fue significativamente mayor en condiciones control en comparación con estrés severo (Tabla 2). Adicionalmente, se observó una disminución significativa en la tasa fotosintética en los genotipos 134 y 148 por efecto del tratamiento de estrés severo. No obstante, el factor de interacción genotipo $\mathrm{x}$ tratamiento no fue significativo (Tabla 2; Figura 3A).

El potencial hídrico foliar fue significativamente más negativo bajo la condición de estrés severo en relación al control y al estrés moderado. Entre estos dos últimos tratamientos no existieron diferencias estadísticamente significativas (Tabla 2; Figura 3B). Dicha respuesta fue dependiente tanto del factor genotipo como por la interacción entre genotipo y tratamiento (Tabla 2). Un comportamiento diferente se registró en el genotipo 10, que mostró diferencias significativas en comparación con todos los genotipos en estudio, ya que mantuvo su potencial hídrico independiente del tratamiento de estrés hídrico aplicado y no disminuyó en respuesta al estrés severo, como se observó en los otros genotipos (Figura 3B).

El análisis de la tasa de crecimiento relativo mostró disminuciones significativas entre los tratamientos de restricción de riego y la interacción tratamiento x genotipo (Tabla 2). De manera contraria, no hubo efecto dependiente del genotipo (Tabla 2). La mayor diferencia se observó entre el control y el estrés severo. Los genotipos 134 y 148 mostraron una tendencia de disminución gradual en la tasa de crecimiento relativa a medida que el tratamiento de estrés fue más severo. El genotipo 93 no mostró diferencias entre control y estrés moderado, pero ante un estrés severo disminuyó dicha tasa de la misma manera que el resto de los genotipos. El genotipo 10 presentó un aumento en la tasa de crecimiento en el tratamiento de estrés leve, pero sin presentar diferencias entre control y estrés severo (Figura 3C).

\section{Discusión}

Mediante la evaluación de parámetros funcionales y de crecimiento, se determinó el nivel de respuesta y desempeño diferencial en cuatro genotipos de vides frente a tratamientos de estrés hídrico. Los genotipos enfrentados a tratamientos de riego deficitario presentaron variaciones contrastantes en los parámetros evaluados. El genotipo 134 fue el que obtuvo un mejor desempeño en términos de sobrevivencia y parámetros de crecimiento tales como longitud de raíz y distancia entrenudos del brote, lo que fue acompañado de una mayor disminución en el intercambio gaseoso y en su potencial hídrico foliar. Esto indicaría una estrategia de tipo anisohídrica, dada tanto por una mayor supervivencia como por promover adaptaciones radiculares, que indicarían un potencial de explorar un mayor 

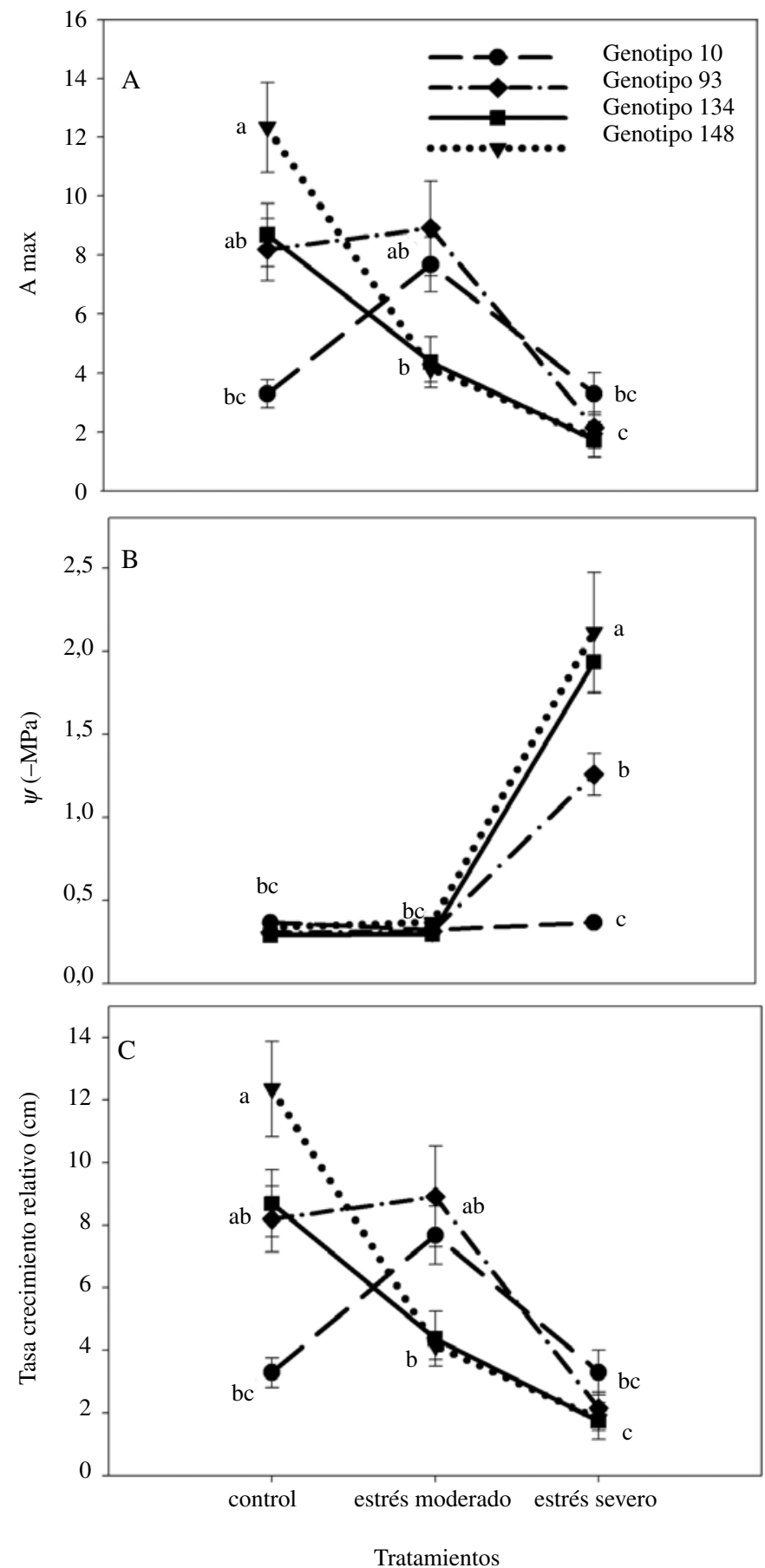

Figura 3. Tasa de fotosíntesis neta (umol $\left.\mathrm{CO}_{2} \mathrm{~m}^{-2} \mathrm{~s}^{-1}\right)(\mathrm{A})$, potencial hídrico foliar ( $\Psi$-MPa) (B) y tasa de crecimiento relativo $(\mathrm{cm})(\mathrm{C})$, medidas en cuatro genotipos diferentes de Vitis vinifera, en condiciones control, estrés hídrico moderado y severo durante tres meses. Se indican los valores promedio ( $\pm 2 \mathrm{SE})$. Letras diferentes corresponden a diferencias significativas; test de Tukey, $\alpha=0,05$.

Net photosynthetic rate (umol $\left.\mathrm{CO}_{2} \mathrm{~m}-2 \mathrm{~s}^{-1}\right)(A)$, leaf water potential $(\Psi-\mathrm{MPa})(\mathrm{B})$ and relative growth rate $(\mathrm{cm})(\mathrm{C})$, assessed in 4 different genotypes of Vitis vinifera, in control, moderate and severe drought stress conditions during three months. Mean values $( \pm 2 S E)$ are shown. Different letters indicate significant differences; Tukey test, $\alpha<0.05$. 
volumen de sustrato, pero adaptando su desempeño fotosintético a la disponibilidad hídrica de suelo (Turner y Jones, 1980; Blum et al., 1989; Schultz, 2003). En un ambiente semiárido, en el cual se conjugan simultáneamente factores ambientales promotores de estrés, como baja disponibilidad hídrica de suelo e incrementos en la demanda hídrica atmosférica, la asimilación de $\mathrm{CO}_{2}$ debe establecer un compromiso entre el gasto hídrico, la fijación de materia y energía y la disipación de la energía excesiva, aplicando los ajustes necesarios para poder mantener la productividad de la vid (Chaves et al., 2010; Cramer, 2010; Lovisolo et al., 2010). Paralelamente, el genotipo 148 fue el que exhibió un comportamiento más dependiente de la disponibilidad de agua suministrada. En la mayoría de los parámetros morfométricos y de desempeño fisiológico presentó un comportamiento similar a las especies sensibles a la sequía (Liakopoulos et al., 2006; Cramer et al., 2007). En contraste, la vid también podría recurrir a un mecanismo de abscisión foliar para poder mantener niveles similares de fotosíntesis en diferentes escenarios de disponibilidad hídrica, con el objetivo de reducir su área de interacción con el ambiente, lo cual de hecho se registró en el genotipo 10 (datos no mostrados).

Aunque se ha sugerido que las diferencias entre cultivares iso- $\mathrm{y}$ anisohídricos pueden incluir diferentes respuestas estomáticas y uso de agua (Schultz, 2003), en general ellos presentan disminuciones similares en parámetros como la conductancia estomática $\left(g_{s}\right)$ y la fotosíntesis máxima en respuesta al estrés hídrico (Lovisolo et al., 2010), lo cual coincide con lo mostrado por los genotipos analizados, en donde se ajustó este carácter fisiológico para ambas estrategias. En efecto, la fotosíntesis máxima varió entre 12 umol $\mathrm{CO}_{2}$ m-2 s-1 en el genotipo 148 y 4 umol CO${ }_{2}$ m-2 s-1 en el genotipo 10 en condiciones control, lo cual es un rango muy amplio entre genotipos de vides a pesar de provenir desde regiones áridas y semiáridas, y cayó bajo 4 umol $\mathrm{CO}_{2} \mathrm{~m}-2$ s-1 en todos los genotipos por efectos del estrés hídrico severo. Tales variaciones entre genotipos son similares a la alta variabilidad encontrada en caracteres fotosintéticos bajo riego entre diferentes cultivares de vid de las islas Baleares, España, a pesar de que estos cultivares comparten un origen común (Bota et al. 2001).

Similarmente, el potencial hídrico fue una respuesta claramente dependiente de los genotipos analizados, en donde el genotipo 10 conservó el potencial hídrico y no disminuyó en respuesta al estrés hídrico severo, a diferencia de los otros genotipos analizados, en donde este potencial se hizo más negativo a medida que aumentó el estrés hídrico. Asimismo, el genotipo 93 presentó un potencial hídrico sobre el umbral-1,5 Mpa en estrés hídrico severo, lo cual indicaría una estrategia isohídrica similar al genotipo 10 (Lovisolo et al., 2010). Este resultado se complementa con una mayor tasa de crecimiento relativo presentado por ambos genotipos en la condición de estrés hídrico moderado, y en particular del genotipo 10 en estrés hídrico severo (Bota et al. 2001; Rogiers et al., 2012). En situaciones de estrés hídrico se requiere una rápida absorción de agua desde el suelo por las raíces, en especial cuando la transpiración es particularmente alta, lo que también puede generar un enorme gradiente de presión hidrostática (Nobel, 2004). Por ello es necesario mantener un potencial hídrico foliar en niveles que permitan mantener un adecuado funcionamiento, sin alterar el desempeño fisiológico.

El estrés hídrico severo inhibió la extensión entrenudos, la expansión foliar y el alargamiento de zarcillos, por lo tanto limita la transpiración, siendo usado como indicador sensible del estado hídrico de la vid (Schultz, 2003; Chaves et al., 2010). Además, disminuciones en el diámetro promedio de los vasos xilemáticos disminuyen la conductividad hidráulica del xilema y pueden prevenir el embolismo, ya que vasos más pequeños son menos susceptibles a la cavitación (Vandeleur et al., 2009; Lovisolo et al., 2010). La respuesta en los genotipos 134 y 148 refleja dicha sensibilidad y adaptación al tratamiento de estrés hídrico moderado, ya que adaptaron la longitud del tallo sin alterar de manera importante la distancia entrenudos, pero con la diferencia de que el genotipo 134 indujo un crecimiento mayor del sistema radical. Esta adaptación del crecimiento y alocación de recursos tallo/raíz permitiría evitar de manera más eficiente los efectos de la cavitación.

\section{Conclusiones}

El sistema experimental de minirizotrones permitió determinar diferencias significativas entre genotipos de vides colectados desde zonas áridas y semiáridas del norte de Chile sometidos a tratamientos de estrés hídrico. Los caracteres morfométricos y atributos de desempeño fisiológico analizados permitieron caracterizar estrategias de respuesta al déficit hídrico, diferenciando entre genotipos anhisohídricos 
como 134 y 148, de genotipos isohídricos como 10 y 93. El conocimiento de los efectos tempranos de la sequía en el desarrollo a nivel de raíces y tallo, adaptaciones de la maquinaria fotosintética y potencial hídrico nos permitió diferenciar los genotipos más tolerantes para su uso potencial como portainjertos o injertos en zonas áridas y semiáridas y/o bajo futuros escenarios de cambios en las condiciones climáticas de la zona norte del país, subrayando el rol fundamental de explotar la variabilidad genética de vides como cultivo modelo.

\section{Agradecimientos}

A.Z.-S. agradece a la carrera de Biotecnología de la Universidad de La Frontera por las facilidades otorgadas para el desarrollo de tesis en nuestro laboratorio. Este artículo contó con el financiamiento y forma parte de las actividades de investigación del proyecto InnovaChile 05CR11PAT-19 a A.Z.-S. Los autores agradecen los valiosos comentarios de los revisores anónimos, quienes permitieron enriquecer el trabajo presentado.

\section{Literatura citada}

Blum, A.; Mayer, J.; Golan, G.

1989 Agronomic and physiological assessments of genotypic variation for drought resistance in sorghum. Australian Journal of Agricultural Research 40: 49-61.

Bota, J.; Flexas, J.; Medrano, H.

2001 Genetic variability of photosynthesis and water use in Balearic grapevine cultivars. Ann Applied Biology 138 (3): 353-361.

Busso, C.

2008 Uso de la cámara de presión y los psicrómetros a termocupla en la determinación de las relaciones hídricas en tejidos vegetales. Phyton (Buenos Aires) 77: 327-350.

Castellarin, S.D.; Pfeiffer, A.; Sivilotti, P.; Degan, M.; Peterlunger,

E.; Di Gaspero, G.

2007 Transcriptional regulation of anthocyanin biosynthesis in ripening fruits of grapevine under seasonal water deficit. Plant, Cell \& Environment 30: 1381-1399.

Cramer, G.R.; Ergül, A.; Grimplet, J.; Tillett, R.; Tattersall, E.; Bohlman, M.; Vincent, D.; Sonderegger, J.; Evans, J.; Osborne, C.; Quilici, D.; Schlauch, K.; Schooley, D.; Cushman, J.

2007 Water and salinity stress in grapevines: early and late changes in transcript and metabolite profiles. Functional \& Integrative Genomics 7: 111-134.

Cramer, G.R.

2010 Abiotic stress and plant responses from the whole vine to the genes. Australian Journal of Grape and Wine Research 16: 86-93.

Chaves, M.M.; Flexas, J.; Pinheiro, C.

2009 Photosynthesis under drought and salt stress: regulation mechanisms from whole plant to cell. Annals of Botany 103: 551-560.

Chaves, M.M.; Zarrouk, O.; Francisco, R.; Costa, J.M.; Santos,

T.; Regalado, A.P.; Rodrigues, M.L.; Lopes, C.M.

2010 Grapevine under deficit irrigation: hints from physiological and molecular data. Annals of Botany 105 (5): 661-76.

Deluc, L.G.; Quilici, D.R.; Decendit, A.; Grimplet, J.; Wheatley, M.D.; Schlauch, K.A.; Mérillon, J.M.; Cushman, J.C.; Cramer, G.R. 2009 Water deficit alters differentially metabolic pathways affecting important flavor and quality traits in grape berries of Cabernet Sauvignon and Chardonnay. BMC genomics 10: 212 .
Flexas, J.; Escalona, J.M.; Medrano, H.

1998 Down-regulation of photosynthesis by drought under field conditions in grapevine leaves. Functional Plant Biology 25: 893-900.

Gaudillère, J.P.; Van Leeuwen, C.; Ollat, N.

2002 Carbon isotope composition of sugars in grapevine, an integrated indicator of vineyard water status. Journal of Experimental Botany 53: 757-763.

Grimplet, J.; Deluc, L.G.; Cramer, G.R.; Cushman, J.C. 2008 Integrating functional genomics with abiotic stress responses in wine grape-Vitis vinifera. in: M. A. Jenks, et al. (eds.), In Advances in Molecular Breeding towards Salinity and Drought Tolerance, Springer Verlag, Holanda, pp. 643-668.

Liakopoulos, G.; Nikolopoulos, D.; Klouvatou, A.; Vekkos,

K.-A.; Manetas, Y.; Karabourniotis, G.

2006 The Photoprotective Role of Epidermal Anthocyanins and Surface Pubescence in Young Leaves of Grapevine (Vitis vinifera). Annals of Botany 98: 257-265.

Lovisolo, C.; Perrone, I.; Carra, A.; Ferrandino, A.; Flexas, J.;

Medrano, H.; Schubert, A.

2010 Drought-induced changes in development and function of grapevine (Vitis spp.) organs and in their hydraulic and non-hydraulic interactions at the whole-plant level: a physiological and molecular update. Functional Plant Biology 37: 98-116.

Morgan, J.M.

1984 Osmoregulation and Water Stress in Higher Plants. Annual Review of Plant Physiology 35: 299-319.

Murashige, T.; Skoog, F.

1962 A revised medium for rapid growth and bioassays with tobacco tissue cultures. Physiology Plant 15: 473-497.

Nobel, P.S.

2004 Physicochemical and Environmental Plant Physiology. Third edition. Academic Press, Inc., San Diego.

Oficina de Estudios y Políticas Agrarias (ODEPA)

2012 Estadísticas de frutales. http://www.odepa.gob.cl/ (consulta en línea).

Rogiers, S.Y.; Greer, D.H.; Hatfield, J.M.; Hutton, R.J.; Clarke,

S.J.; Hutchinson, P.A.; Somers, A.

2012 Stomatal response of an anisohydric grapevine cultivar to evaporative demand, available soil moisture and abscisic acid. Tree Physiol. 32 (3): 249-61. 
Schultz, H.R.

2003 Differences in hydraulic architecture account for near isohydric and anisohydric behaviours of two field-grown Vitis vinifera L. cultivars during drought. Plant, Cell \& Environment 26, 1393-1405.

Sokal, R.; Rohlf, F.J.

1995 Biometry: the principles and practice of statistics in biological research Freeman Company, Nueva York.

Tattersall, E.; Grimplet, J.; DeLuc, L.; Wheatley, M.; Vincent, D.; Osborne, C.; Ergül, A.; Lomen, E.; Blank, R.; Schlauch, K.; Cushman, J.; Cramer, G.

2007 Transcript abundance profiles reveal larger and more complex responses of grapevine to chilling compared to osmotic and salinity stress. Functional \& Integrative Genomics 7: 317-333.

Turner, N.C.; Jones, M.M.

1980 Turgor maintenance by osmotic adjustment: a review and evaluation, in: N. C. Turner and P. J. Kramer (eds.), Adaptation of plants to water and high temperature stress, Wiley Inter Science, Nueva York. pp. 84-104.

Vandeleur, R.K.; Mayo, G.; Shelden, M.C.; Gilliham, M.; Kaiser, B.N.; Tyerman, S.D.

2009 The role of PIP aquaporins in water transport through roots: diurnal and drought stress responses reveal different strategies between isohydric and anisohydric cultivars of grapevine. Plant Physiology 149:445-460. 